\title{
Integrating Incoming Information into Discourse Model in Tunisian Arabic
}

Marwa Mekni Toujani

Higher Institute of Languages of Tunis, University of Carthage, Tunisia

https://doi.org/10.36505/ExLing-2017/08/0028/000330

\begin{abstract}
The endeavor of the present study is to gauge the time course of connecting incoming information to information mentioned earlier in the text that are no longer available in Working Memory (WM). Two word-by-word self-paced reading experiments based on the contradiction paradigm were used. The Group Embedded Figure Test (GEFT) was utilized to explore participants' field-dependency. Data from 96 Tunisians reveals that reading times in the inconsistent condition were higher than the consistent condition in the post-critic region when participants were instructed to adopt the protagonist point of view. However, this difference did not reach the significance level when participants were instructed to read for mere comprehension. The results also reveal that reading times for field-dependent participants were higher than their field-independent counterparts. Thus, this study yields evidence for the dominance of local coherence over global coherence.
\end{abstract}

Key words: Discourse processing, time-course, field-dependency

\section{Introduction}

There are two main lines in discourse processing research. The first one is interested in understanding the type of inferences that constitute discourse representations (Stewart, Kidd, \& Haigh, 2009). The second line is interested in the time course of integrating incoming information with the unfolding discourse model (ibid). Incoming information can be integrated as soon as it is available or it is integrated at spillover regions as a wrap-up effect (Guzman \& Klin, 2000). This study addresses the second line of research and aspires to answer the following questions: (1) does readers' sensitivity to spatial anomaly affect the time-course of integrating incoming information into the unfolding discourse model in Tunisian Arabic (TA)? (2) do field dependency and task demands affect the timecourse of integration in TA?

\section{Experimental methodology}

The TA version of Stewart et al.'s (2009) sixteen experimental texts were used to explore the phenomenon of time-course of discourse processing in reading. The texts were translated by the researcher, were checked by an expert, and were finally piloted to check their "naturalness", their coherence and the familiarity of the topics. The texts were developed according to the contradiction paradigm (O’Brien \& Albrecht, 1992).

ExLing 2017: Proceedings of 8th Tutorial and Research Workshop on Experimental Linguistics, 19-22 June, Heraklion, Crete, Greece 
The two non-cumulative center word-by word present experiments were run using PsychoPy2 software (Pierce, 2009) on a laptop. Participants were asked to read texts at their normal pace in order to respond to comprehension questions at the end of each passage (experiment 1) and to adopt the protagonist point of view (Experiment 2).

Each text is composed of eight sentences. The first sentence is an introduction mentioning the spatial location. The second, the third and the fourth sentences are filler materials. The fifth sentence is the target sentence. The sixth sentence is the post-target one. The seventh and the eighth sentences are the concluding sentences. There were two versions of each passage. Each passage appeared once in each of the two conditions (consistent or inconsistent condition). Each passage in both experiments was followed by a yes/no comprehension question.

Besides, the GEFT (Witkin, Raskin, Oltman, \& Karp, 1971).was utilized to gauge participants field dependency. The GEFT scores ranged from zero to 17 in this study. Participants were categorized as field-dependent (FD) or field-independent (FI) based on the median split option (Raptis, Fidas, \& Avouris, 2016). Participants whose scores ranged from zero to five were considered as FD. Participants whose scores ranged from six to 17 were considered as FI.

Ninety-six Tunisian third-year University students of English with native language TA participated in the study (48 participants per experiment).

\section{Results}

The total time (in milliseconds) that participants took to read was analyzed for each region. (1) The critical region consists of lexical items that convey the location information. (2) The material following the location items until the end of the target sentence represents the post-critical region. (3) The post-target sentence is the following sentence. Repeated measure ANOVA with both region and condition as within-subjects factors, field dependency as a between-subjects factor and reading times as a dependent variable was used. Both analysis by subjects and analysis by items as random factors were implemented. All analyses reported were significant at the .05 alpha level.

\section{Experiment 1}

There was no significant effect of condition in the three regions. However, there was a main effect of field-dependency F1 $(1,46)=9.148, \mathrm{p}=.004$ with FD participants spent more times reading the texts in both conditions $(\mathrm{M}=$ 2850.242, STD $=125.603)$ than FI participants did $(\mathrm{M}=2326.513, \mathrm{STD}=$ 115.538). Comprehension question response accuracy was $92 \%$. 


\section{Experiment 2}

For the post-critical region, there was a significant effect of condition for item analysis $\mathrm{F} 2(1,15)=7.284, \mathrm{p}=.01$ with this region is more slowly read in the inconsistent condition $(\mathrm{M}=2783.62$, STD =1604.656) than the consistent condition $(\mathrm{M}=2362.69, \mathrm{STD}=1235.421)$. However, for analysis by participants, the difference between inconsistent and consistent condition only approached significance level $F 1(1,46)=2.843, p=.09$. For both the critical region and the post-target region, there was no effect of condition. There was also no main effect of field-dependency F1<1. Comprehension question response accuracy was $95 \%$.

\section{Discussion and conclusions}

The results of the first experiment were in line with McKoon and Ratcliff's (1992) Minimalist Theory. That is, only is information essential for establishing local coherence (connecting the incoming information to one or two prior sentences) is integrated during reading. Global coherence is only maintained online when there is a local coherence break or when readers are instructed to do so (ibid).

Based on the results of the second experiment, integrating incoming information into discourse model is delayed as it is a part of a wrap-up operation. This result is constant with Guzman and Klin's (2000) results. However, this delayed online integration only occurs when participants were instructed to adopt the protagonist point of view. Again, this result endorses the Minimalist Approach.

As the spatial information was the one manipulated in the present experiments, the results are basically in line with Smith and O'Brien's (2012) results. As they argued, participants do not track spatial information if they are not instructed to do so. This instruction triggered their deep processing.

Hence, a revisited version of Cook and Myers (2004) two-stage framework can be suggested. The first stage consists of linking the information at hand with information that is in WM or that is easily accessed from long-term memory (McKoon \& Ratcliff, 1992). This stage is automatic and occurs no matter the conditions are. The second stage only occurs when readers have special goals aiming at deep processing to meet their high standards of coherence (Van den Broek, Bohn-Gettler, Kendeou, Carlson, \& White, 2011). This stage occurs at wrap-up positions. In future research, protagonist characteristics will be manipulated in order to deepen our understanding of the time-course of discourse processing.

Based on the results of both experiments, it can be noticed that FI participants reacted faster to inconsistent materials than FD ones did, which 
supports Hannon and Daneman (2001) who argue that FD people have difficulty in detecting anomalies. More interestingly, the effect of field dependency on the time course of text processing was minimized when participants were instructed to engage in an activity that requires deep processing (i.e. adopting the protagonist point of view). In fact, task demands have a primacy effect over individual differences.

\section{Acknowledgment}

The Experiments were conducted in partial fulfillment of the requirements for my $\mathrm{PhD}$ degree conducted under the direction of Pr. Mohamed Jabeur to whom go my deepest thanks. I am also grateful to Dr. Tarek Hermessi for helping me reshaping the topic and to Dr. Hafedh Halila for editing the translated materials.

\section{References}

Cook, A.E., Myers, J.L. 2004. Processing discourse roles in scripted narratives: The influences of context and world knowledge. Journal of Memory and Language, 50, 268-288.

Guzman, A.E., Klin, C.M. 2000. Maintaining global coherence in reading: The role of sentence boundaries. Memory \& Cognition, 28, 722-730.

Hannon, B., Daneman, M. 2001. A new tool for measuring and understanding individual differences in the component processes of reading comprehension. Journal of Educational Psychology, 93, 103e128.

McKoon, G., Ratcliff, R. 1992. Inference during reading. Psychological Review, 99, 440-466.Smith, E.R., O’Brien, E.J. 2012. Tracking spatial information during reading: A cue-based process. Memory \& Cognition, 40, 791-801.

O'Brien, E.J., Albrecht, J.E. 1992. Comprehension strategies in the development of a mental model. Journal of Experimental Psychology: Learning, Memory, and Cognition, 18, 777-784. doi:10.1037/0278-7393.18.4.777

Peirce, J.W. 2009. Generating stimuli for neuroscience using PsychoPy. Front. Neuroinform. 2:10. doi:10.3389/neuro.11.010.2008

Raptis, G., Fidas, C., Avouris, N. 2016. Differences of field dependent/independent gamers on cultural heritage playing: Preliminary findings of an eye-tracking study. In Proceedings of International Conference on Digital Heritage (Euromed2016), Nicosia, Cyprus, 2016. Springer. doi:10.1007/978-3-319-48974- 2_22

Smith, E.R., O'Brien, E.J. 2012. Tracking Spatial Information during Reading: A Cue-Based Process. Memory \& Cognition, 40, 791-801.

Stewart, A.J., Kidd, E., Haigh, M. 2009. Early Sensitivity to Discourse-Level Anomalies: Evidence From Self-Paced Reading. Discourse Processes,46:1,46 69. DOI: 10.1080/01638530802629091

Van den Broek, P., Bohn-Gettler, C., Kendeou, P., Carlson, S., White, M. J. 2011. When a reader meets a text: The role of standards of coherence in reading comprehension. In M.T. McCrudden, J.P. Magliano, G. Schraw (Eds.), Text relevance and learning from text (pp. 123-140). Greenwich, CT: Information Age

Witkin, H.A., Raskin E., Oltman, P.K., Karp, S.A. (1971). A manual for the Group Embedded Figures Test. Palo Alto, CA: Consulting Psychologists Press. 